\title{
ROLE OF MEDIA IN OVERALL DEVELOPMENT OF THE COUNTRY: AN ANALYSIS
}

\section{VIRENDRA KUMAR SAINI AND VARUN KUMAR}

Department Of Commerce, Government PG College Jaiharikhal, Lansdowne, Uttarakhand

*Corresponding Author Email: varsh21210907@gmail.com

Received: 12.11.2020; Revised: 10.12.2020; Accepted: 24.12.2020

CSociety for Himalayan Action Research and Development

\begin{abstract}
For the development of any nation, progressive ideological change in society is very important. Only through communication this change can be brought in the society and economic, social and political development of the nation can be ensured. Today in the changing world system in the form of this global village, India is the second largest country to use telecom services. The presented research paper describes the progressive role of media in the development of the nation.
\end{abstract}

Keywords: National Development, Role of Media, Analysis

\section{देश के समग्र विकास में संचार माध्यमों की भूमिका-एक विश्लेषण \\ वीरेंद्र कुमार सैनी एवं वरूण कुमार वाणिज्य विभाग}

राजकीय स्नातकोत्तर महाविद्यालय, जयहरीखाल, पौडी गढ़वाल mail- agmsaini1981@gmail.com

सारांश

किसी भी राष्ट्र के विकास के लिए समाज में प्रगतिशील वैचारिक परिवर्तन अति आवश्यक है और संचार के माध्यम से ही समाज में वैचारिक परिवर्तन लाया जा सकता है और राष्ट्र का सामाजिक, आर्थिक, राजनीतिक, विकास सुनिश्चित हो सकता है। आज इस वैश्विक ग्राम के रूप में परिवर्तित होती विश्व व्यवस्था में भारत राष्ट्र दूरसंचार माध्यमों का उपयोग करने वाला विश्व का दूसरा सबसे बड़ा देश बन गया है। प्रस्तुत शोध पत्र में राष्ट्र के विकास में संचार माध यमों की भूमिका एक प्रगतिशीलता का एक अध्ययन प्रस्तुत किया गया है।

कुंजी शब्द: राष्ट्रीय विकास, मीडिया की भूमिका, विश्लेषण 


\section{प्रस्तावना}

भारतीय परिदृश्य में प्राचीन काल से ही संचार हर समुदाय की प्राथमिक आवश्यकता बन चुका था। प्राचीन काल में आदिमानव संकेत और ध्वनि का प्रयोग कर संचार करते थे उसके उपरांत गुफा मैंचित्रण भी संचार का ही एक माध्यम था। विभिन्न परंपराओं के विकास के साथ ही देश में फैली हुई कुरीतियों और अंधविश्वासों को परंपरागत संचार माध्यम चित्रकला, लोक नृत्य, लोकगीत लोक, कथा लोकनाट्य, कठपुतली, चिन्ह आकृतियों के माध्यम से वैचारिक परिवर्तन कर अधिकांश कुरीतियों जैसे बाल विवाह, बहुविवाह, सती प्रथा, जनसंख्या विस्फोट, निरक्षरता, नशा , कुपोषण, जाति प्रथा, दहेज प्रथा आदि को समाज से पृथक किया। देश में आजादी के आंदोलन में भी जन जागरण हेतु विभिन्न संचार माध्यमों जैसे लोकनाटक, लोकगीत, नौटंकी, स्थानीय समाचार पत्र पत्रिका प्रभात फेरिया का प्रयोग कर आंदोलन की लौ को जला कर रखा है। संचार के सामाजिक महत्व के साथ साथ आज इस पर राजनीतिक और आर्थिक विकास की धुरी टिकी हुई है। इन बिंदुओं पर ही समाज और समुदाय विकसित होते हैं। इसलिए संचार का महत्व और भी बढ़ जाता है क्योंकि किसी भी राष्ट्र की भौगोलिक सीमाएं उसके विकास में बाधा नहीं बन सकती हैं। संचार माध्यमों से आज दूर बैठे विभिन्न राष्ट्रों से मेलमिलाप आर्थिक गतिविधियो तथा वैश्विक समस्याओं का दैनिक निदान संभव हो पाया है और वैश्विक ग्राम परिकल्पना सिद्ध प्रतीत होती है।

गिन्सवर्ग के अनुसार-" जनता असंगठित और अनाकार व्यक्तियों का समूह है, जिसके सदस्य सामान्य इच्छाओं एवं मतों के आधार पर एक दूसरे से बंधे रहते हैं परंतु इसकी संख्या इतनी बड़ी होती है कि वे एक-दूसरे के साथ प्रत्यक्ष रूप से व्यक्तिगत संबंध नहीं रख सकते हैं।"

रॉबर्ट एंडरसन ने परिभाषित किया है कि-"वाणी, लेखन या संकेतों के द्वारा विचारों, अभीमतों अथवा सूचना का विनिमय करना संचार कहलाता है।"

\section{संचार माध्यमों के विभिन्न प्रकार हैं-}

(क) व्यक्ति परक / परंपरागत संचार माध्यम- चित्रकला, लोकनृत्य, लोक गीत, लोक कथा, कठपुतली आदि।

(ख) यांत्रिक / आधुनिक संचार माध्यम।

(ख1) मुद्रित संचार माध्यम-समाचार पत्र, पत्र पत्रिकाएं, ब्रोशर, पोस्टर, होर्डिंग आदि।

(ख2) दूरसंचार माध्यम- रेडियो, टेलीविजन, सिनेमा, उपग्रह संचार, टेलीग्राम, टेलीप्रिंटर, फैक्स मशीन, टेलीफोन, रेडियो पेजिंग, वॉकी टॉकी, इंटरनेट (फेसबुक, यूट् यूब, इंस्टाग्राम, ट्विटर आदि)

\section{शोध पत्र में प्रयुक्त कार्यविधि-}

प्रस्तुत अध्ययन द्वितीयक आंकडों पर आधारित है।

\section{आंकड़ों का प्रस्तुतीकरण}

भारत में संचार माध्यम के विकास का तुलनात्मक अध्ययन जिससे स्वतः स्पष्ट होता है कि किस प्रकार संचार माध्यमों का अनवरत विकास हुआ और लैंडलाइन की तुलना में मोबाइल की विभिन्न उपयोगिता के कारण उपभोक्ताओं की संख्या में अप्रत्याशित वृद्धि हुई।

तालिका-1 में प्रस्तुत तुलनात्मक अध्ययन से स्पष्ट होता है कि वर्ष 2001 के सापेक्ष वर्ष 2019 तक मोबाइल फोन उपभोक्ताओं की संख्या में 1158.13 मिलियन उपभोक्ताओं की वृद्धि हुई है और लैंडलाइन फोन के सापेक्ष मोबाइल फोन अपनी अनेकों उपयोगिता के कारण शहरी और ग्रामीण दोनों ही परिवेश स्वीकार किया गया। 
Himalayan J. Soc. Sci. \& Humanities ISSN: 0975-9891

Vol. 15, (2020) 49-53

DOI: https://doi.org/10.51220/hjssh.v15i1.6

तालिका 1 - लैं डलाइन और मोबाइल फोन उपभोक्ताओं की संख्या सारणी (2001-2019) ( डी.ओ.टी)

\begin{tabular}{|c|c|c|}
\hline प्रतिवर्ष माह मार्च में & $\begin{array}{c}\text { मोबाइल फोन उपभोक्ताओं की संख्या } \\
\text { (मिलियन में) }\end{array}$ & $\begin{array}{c}\text { लैंडलाइन फोन उपभोक्ताओं की संख्या } \\
\text { (मिलियन में) }\end{array}$ \\
\hline 2001 & 3.58 & 32.70 \\
\hline 2002 & 6.68 & 38.29 \\
\hline 2003 & 13.29 & 41.32 \\
\hline 2004 & 35.62 & 40.92 \\
\hline 2005 & 56.95 & 41.42 \\
\hline 2006 & 101.87 & 40.22 \\
\hline 2007 & 165.09 & 40.77 \\
\hline 2008 & 261.08 & 39.41 \\
\hline 2009 & 391.76 & 37.96 \\
\hline 2010 & 584.32 & 36.96 \\
\hline 2011 & 811.6 & 34.73 \\
\hline 2012 & 919.18 & 32.17 \\
\hline 2013 & 867.81 & 30.21 \\
\hline 2014 & 904.52 & 28.50 \\
\hline 2015 & 969.54 & 26.59 \\
\hline 2016 & 1034.11 & 25.22 \\
\hline 2017 & 1170.59 & 24.40 \\
\hline 2018 & 1188.99 & 22.81 \\
\hline 2019 & 1161.71 & 21.70 \\
\hline
\end{tabular}

स्रोत- डी.ओ.टी (कंपाइल्ड डाटा)

तालिका 2- भारत देश में इंटरनेट उपभोक्ताओं तुलनात्मक अध्ययन से स्पष्ट होता है कि शहरी परिवेश में ही नहीं अपितु ग्रामीण परिवेश में भी इंटरनेट जनसंचार माध्यम का अत्यधिक उपयोग पिछले कुछ वर्षो में बढ़ा है।

तालिका 2- ग्रामीण और शहरी इंटरनेट उपभोक्ताओं का तुलनात्मक अध्ययन (2015-2019)

\begin{tabular}{|l|l|l|}
\hline प्रतिवर्ष माह मार्च में & $\begin{array}{l}\text { ग्रामीण उपभोक्ताओं की संख्या } \\
\text { (मिलियन में) }\end{array}$ & $\begin{array}{l}\text { शहरी उपभोक्ताओं की संख्या (मिलियन } \\
\text { में) }\end{array}$ \\
\hline 2015 & 107.56 & 194.77 \\
\hline 2016 & 111.95 & 230.71 \\
\hline 2017 & 136.52 & 285.68 \\
\hline 2018 & 145.83 & 348.13 \\
\hline 2019 & 227.01 & 409.72 \\
\hline
\end{tabular}

स्रोत ट्राई 
उपरोक्त अध्ययन से स्पष्ट होता है कि वर्तमान बदलते परिवेश में संचार के महत्व को पहचान कर जनमानस ने उसको स्वीकार किया, वह विभिन्न सरकारों के द्वारा संचार माध्यम को प्रोत्साहित किया गया, जिस कारण उसकी पहुंच एक आमजन तक हुई, और डिजिटल इंडिया का सपना देखा गया। उसकी उपयोगिता के महत्व को देखते हुए सरकार के द्वारा एक नई दूरसंचार नीति 2018 लाई गई, जिससे संचार माध्यमों का और अत्यधिक विकास कर उसका उपयोग संपूर्ण राष्ट्र के सामाजिक आर्थिक राजनीतिक क्षेत्र में किया जा सके। आज प्रौद्योगिकी के क्षेत्र में भारत देश विश्व के विकसित देशों की पंक्ति में खडा है और यह सुदृढ़ संचार व्यवस्था से ही संभव हुआ है।

संचार व्यवस्था के कारण ही समाज कल्याण सुनिश्चित हो पाया है। आज समाज के विभिन्न कमजोर, दिव्यांग, वृद्धजन आदि को सरकार के द्वारा प्रदान की जाने वाली योजनाओं का लाभ पारदर्शिता के साथ पहुंचा है, जिससे उनका जीवन स्तर सुधरा है। इसका जीवंत उदाहरण कोविड-19 वैश्विक महामारी में संचार साधनों का प्रयोग कर संपूर्ण समाज में जन जागरण कर महामारी से बचने के लिए अद्यतन जानकारी को साझा किया जा रहा है। संचार का प्रयोग कर ही आरोग्य सेतु ऐप को लांच किया गया वहीं किसी भी जानकारी के लिए दूरभाष नंबर 1075 जारी किया गया। यह सब संचार से ही संभव हो पाया है अन्यथा ज्ञान के अभाव में यह वैश्विक महामारी विकराल रूप में बदल जाती इसी प्रकार समाज के अन्य वर्गों के लिए भी भारत सरकार के द्वारा हैल्पलाइन नंबर जारी किए गए उदाहरणार्थ चाइल्ड हेल्पलाइन नंबर 1098, महिला हेल्पलाइन नंबर 1091, मिसिंग चाइल्ड एंड वूमेन हेल्पलाइन नंबर 1094, नेशनल इमरजेंसी नंबर 112, पुलिस सहायता नंबर 100, सीनियर सिटीजन हेल्पलाइन नंबर 1291 आदि जिससे समाज को एक नई दिशा मिली है। यह सभी संचार का एक उदाहरण है, जिससे यह सब संभव हो पाया।

शिक्षा के संदर्भ में दूर संचार माध्यम से क्रांति आई है। आज शिक्षा किसी राष्ट्र की भौगोलिक सीमाओं से बंधी नहीं है आज एक स्थान से विश्व् के किसी भी बड़े शिक्षा संस्थान आसानी से संपर्क स्थापित कर शिक्षा ग्रहण की जा सकती है। वर्तमान समय में विश्व में फैली वैश्विक महामारी कोविड-19 ने संस्थागत शिक्षा को प्रभावित किया है। लेकिन दूर संचार माध्यमों ने शिक्षा के क्षेत्र में भी अपनी उपस्थिति सिद्ध करती है। आज ऑनलाइन कक्षाओं के माध्यम से अधिकांश शिक्षण संस्थान अपना शिक्षण कार्य कर पा रहे हैं। दूसरी ओर आज बहुत सी ऑनलाइन वेबसाइट है जिन पर ई बुक, ई लाइब्रेरी जैसी समस्त पाठ्यक्रम पुस्तके उपलब्ध हैं। आज भारत सरकार के द्वारा शिक्षा को बढ़ावा देने के लिए कहीं दूरदर्शन कार्यक्रम स्वयं प्रभा के माध्यम से दूरदराज के क्षेत्रों में बैठे अभ्यर्थियों को लाभान्वित किया है। यह सब कुछ सुदृढ़ संचार व्यवस्था से संभव हो पाया है।

आर्थिक क्षेत्र का संचार माध्यमों ने स्वरूप ही बदल दिया है। परंपरागत बाजारों की संस्कृति बदल गई है। परंपरागत विश्व बाजार ई बाजार में तब्दील हो गया है। ऑनलाइन माध्यम से खरीददारी में अप्रत्याशित वृद्धि हो रही है। आज घर बैठे विश्व के किसी भी कोने से अपनी इच्छा के अनुसार कोई भी वस्तु सेवा दी जा सकती है। रेल सेवा, बस सेवा, हवाई सेवा आदि को सरलता से बुक किया जा सकता है। आज विश्व् की बड़ी-बड़ी कंपनियां ऑनलाइन बाजार में अपना वर्चस्व सिद्ध करने में लगी हैं। आज विश्व को भारत में सबसे बड़ा बाजार दिखाई देता है और यह सब संचार माध्यम के कारण ही संभव हो पाया है।

निष्कर्ष- आज भारतीय राष्ट्र की समग्र विकास की धुरी संचार पर ही टिकी है। संचार के माध्यम पत्रकारिता को चतुर्थ स्तंभ के रूप में स्वीकार किया गया है। जिसके द्वारा संसद के तीनों स्तंभों न्यायपालिका, विधायिका और कार्यपालिका की समीक्षा कर एक सजग प्रहरी की भूमिका निभाई है। दूसरी ओर जनतंत्र की वाक और अभिव्यक्ति की स्वतंत्रता की रक्षा की है। वहीं विकास के समस्त आयाम शिक्षा, स्वास्थ्य, बैंकिग, ई व्यापार, ई प्रशासन, मीडिया, प्रौद्योगिकी, उपग्रह विकास सुनिश्चित किया है वहीं ग्रामीण भारत की बदलती तस्वीर भी किसी से छिपी नहीं है। आर्थिक रूप से सुदृढ़ होते राष्ट्र की वर्ष 2017 की जीडीपी में .76 प्रतिशत हिस्सेदारी ई व्यापार की रही है, जिसमें निरंतरवृद्धि हो रही है। वर्ष 2014 में व्यापार का क्षेत्र 14 बिलियन यू०एस० डॉलर का था जो वर्ष 2020 में बढ़कर 64 बिलियन डॉलर हो गया है। इससे मेक इन 
इंडिया का सपना, डिजिटल इंडिया का सपना सच होता नजर आ रहा है। आज भारत राष्ट्र में 346.2 मिलि यन फेसबुक यूजर्स हैं और साप्ताहिक प्रकाशित होने वाले अखबारों की संख्या 105443 है। विकसित होते संचार माध्यमों के कारण संपूर्ण विश्व एक परिवार सदृश्य वैश्विक ग्राम बना गया है और वसुधेव कुटुंबकम की भावना फलीभूत होने लगी है। सचमुच आज सूचना संचार और मनोरंजन की दुनिया मे अभूतपूर्व चमत्कार हो रहा है। इससे जीवन की गुणवत्ता प्रभावित हो रही है और एक न या अर्थ तंत्र पनप रहा है जिसकी पहुंच का क्षेत्र निरंतर बढ़ता ही जा रहा है उसे हर क्षेत्र में ज्यादा से ज्यादा विकल्प मिल रहे हैं। सूचना का संसार हमारे जीवन में छा रहा है और इसके परिणाम अपरिभाषित हैं यह कहना कोई अतिशयोक्ति नही होगी कि राष्ट्र विकास और संचार के बीच एक सकारात्मक से सम्बन्ध हैं। वर्तमान युग में बिना संचार के राष्ट्र विकास संभव नही है।

\section{संदर्भ ग्रंथ सूची-}

संचार मंत्रालय भारत सरकार नई दिल्ली के द्वारा प्रकाशित वार्षिक रिपोर्ट 2017-2018

दूरसंचार विभाग द्वारा प्रकाशित टेलीकॉम स्टैटिसटिक्स इंडिया 2019

डिपार्टमेंट ऑफ टेलीकम युनिकेशन वेबसाइट www.dot.gov.in

वेबसाइट इण्डि यन हेल्पलाइन डॉट कॉम www.indianhelpline.com

वेबसाइट www.statista.com 